\title{
LA-UR-16-23421
}

Approved for public release; distribution is unlimited.

Title: SE Requirements Development Tool User Guide

Author(s): $\quad$ Benson, Faith Ann

Intended for: $\quad$ Report

Issued: 
Disclaimer:

Los Alamos National Laboratory, an affirmative action/equal opportunity employer, is operated by the Los Alamos National Security, LLC for the National Nuclear Security Administration of the U.S. Department of Energy under contract DE-AC52-06NA25396. By approving this article, the publisher recognizes that the U.S. Government retains nonexclusive, royalty-free license to publish or reproduce the published form of this contribution, or to allow others to do so, for U.S. Government purposes. Los Alamos National Laboratory requests that the publisher identify this article as work performed under the auspices of the U.S. Department of Energy. Los Alamos National Laboratory strongly supports academic freedom and a researcher's right to publish; as an institution, however, the Laboratory does not endorse the viewpoint of a publication or guarantee its technical correctness. 


\section{SE Requirements Development Tool \\ User Guide}

August 2015

Version 1.0

\section{Los Alamos National Laboratory}

Operated by Los Alamos National Security, LLC, for the U.S. Department of Energy's NNSA (c) 2015 LANS, LLC. All rights reserved. 
This tool is based on information contained in the January 2001 Systems Engineering Fundamentals Guide prepared by the Defense Acquisition University Press, and the INCOSE Systems Engineering Handbook, v. 3.2, Jan. 2010

Operated by Los Alamos National Security, LLC, for the U.S. Department of Energy's NNSA (c) 2015 LANS, LLC. All rights reserved. 


\section{Document Revisions}

\begin{tabular}{l|l|l}
\multicolumn{1}{c|}{ Date } & \multicolumn{1}{|c}{$\begin{array}{c}\text { Version } \\
\text { Number }\end{array}$} & Document Changes \\
\hline $08 / 03 / 2015$ & 0.1 & Initial Draft \\
\hline & & \\
\hline & & \\
\hline & & \\
\hline & & \\
\hline & & \\
\hline & &
\end{tabular}




\section{Table of Contents}

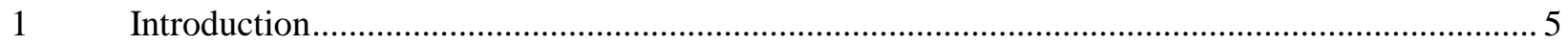

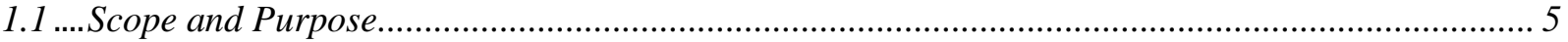

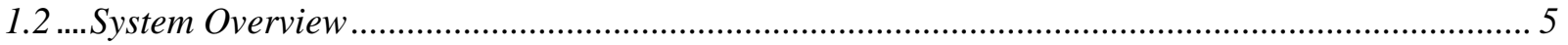

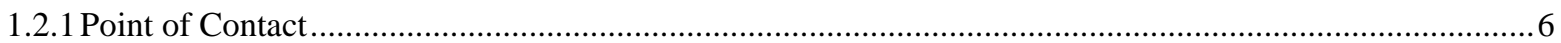

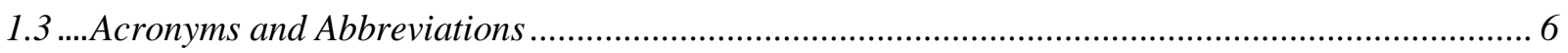

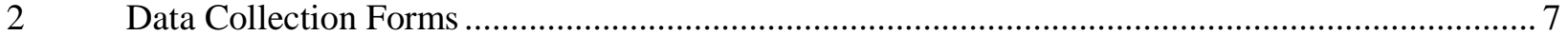

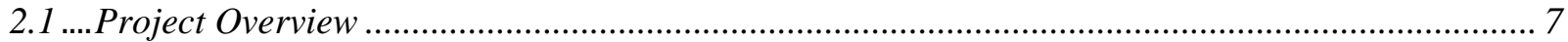

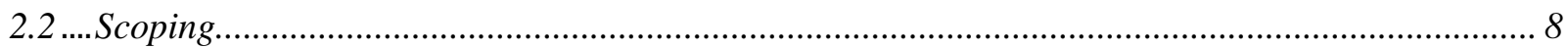

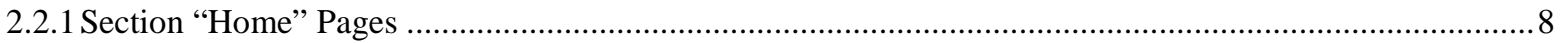

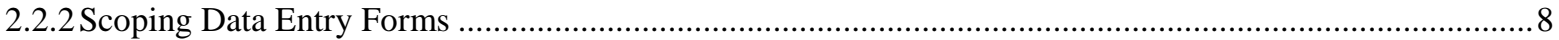

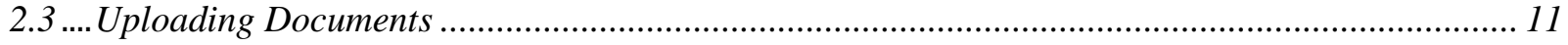

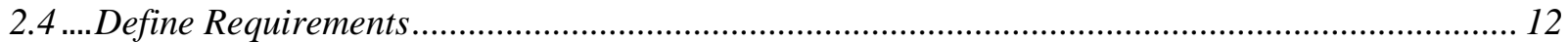

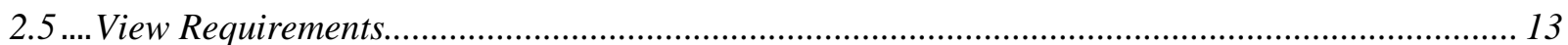

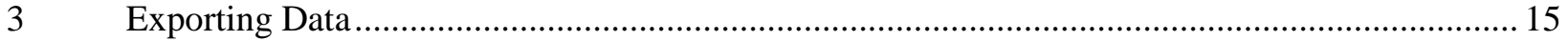




\section{Introduction}

\subsection{Scope and Purpose}

The LANL Systems Engineering Requirements Development Tool (SERDT) is a data collection tool created in InfoPath for use with the Los Alamos National Laboratory's (LANL) SharePoint sites. Projects can fail if a clear definition of the final product requirements is not performed. For projects to be successful requirements must be defined early in the project and those requirements must be tracked during execution of the project to ensure the goals of the project are met. Therefore, the focus of this tool is requirements definition. The content of this form is based on International Council on Systems Engineering (INCOSE) and Department of Defense (DoD) process standards and allows for single or collaborative input. The "Scoping" section is where project information is entered by the project team prior to requirements development, and includes definitions and examples to assist the user in completing the forms. The data entered will be used to define the requirements and once the form is filled out, a "Requirements List" is automatically generated and a Word document is created and saved to a SharePoint document library. SharePoint also includes the ability to download the requirements data defined in the InfoPath from into an Excel spreadsheet.

This User Guide will assist you in navigating through the data entry process.

NOTE: The SERDT is designed to open in a web browser using Internet Explorer. Using other browsers will work but may result in errors and loss of functionality.

\subsection{System Overview}

The SERDT is accessed via the SharePoint site:

https://ade.lanl.gov/aet/FBDev1/SERequirementsTool_New

However, access to the SharePoint server is restricted. To request access, send an email to Faith Benson at fbenson@lanl.gov.

The SERDT contains three main levels of data collection and also allows for uploading supporting documentation. The three main levels of data collection include:

1. Overall project information: Project Overview - This section focuses on the overarching project need high-level goals.

2. Scoping information: Scoping Section - This section focuses on defining the work scope and deliverables of the project as well as any predefined expectations or constraints. User inputs should focus on "what" the project needs to accomplish.

3. Requirements definition: Requirements Definition section - This section is used to define specific, measurable requirements (how the project needs will be met) as well as any predefined specific project constraints or customer expectations.

Once the information is collected and requirements defined, a list of requirements is automatically generated and exported into a Word document, which is then saved to a separate SharePoint document library. This is an automated process. The user is not required to do 


\section{- Los Alamos SE Requirements Development ToO USER GUDE}

anything other than enter the data. The user can also export the defined requirements into and Excel spreadsheet.

\subsubsection{Point of Contact}

For assistance, contact Faith Benson at 665-7299.

\subsection{Acronyms and Abbreviations}

DE Data Entry

DoD Department of Defense

INCOSE International Council on Systems Engineering

LANL Los Alamos National Laboratory

SE $\quad$ Systems Engineering

SERDT Systems Engineering Requirements Development Tool 


\section{Data Collection Forms}

\subsection{Project Overview}

The Project Overview is the first form in the SERDT. Here you will enter top-level information about your project, including:

1. Date

2. Project Name

3. Customer - The organization requesting the work

4. Project Lead and LANL Participants - click the people picker Help button for more information on entering Project Lead and LANL participants. The "Assign and Notify" functionality is in development.

5. Non-LANL Participants (does not work with people picker).

6. Location Information

7. Project Summary

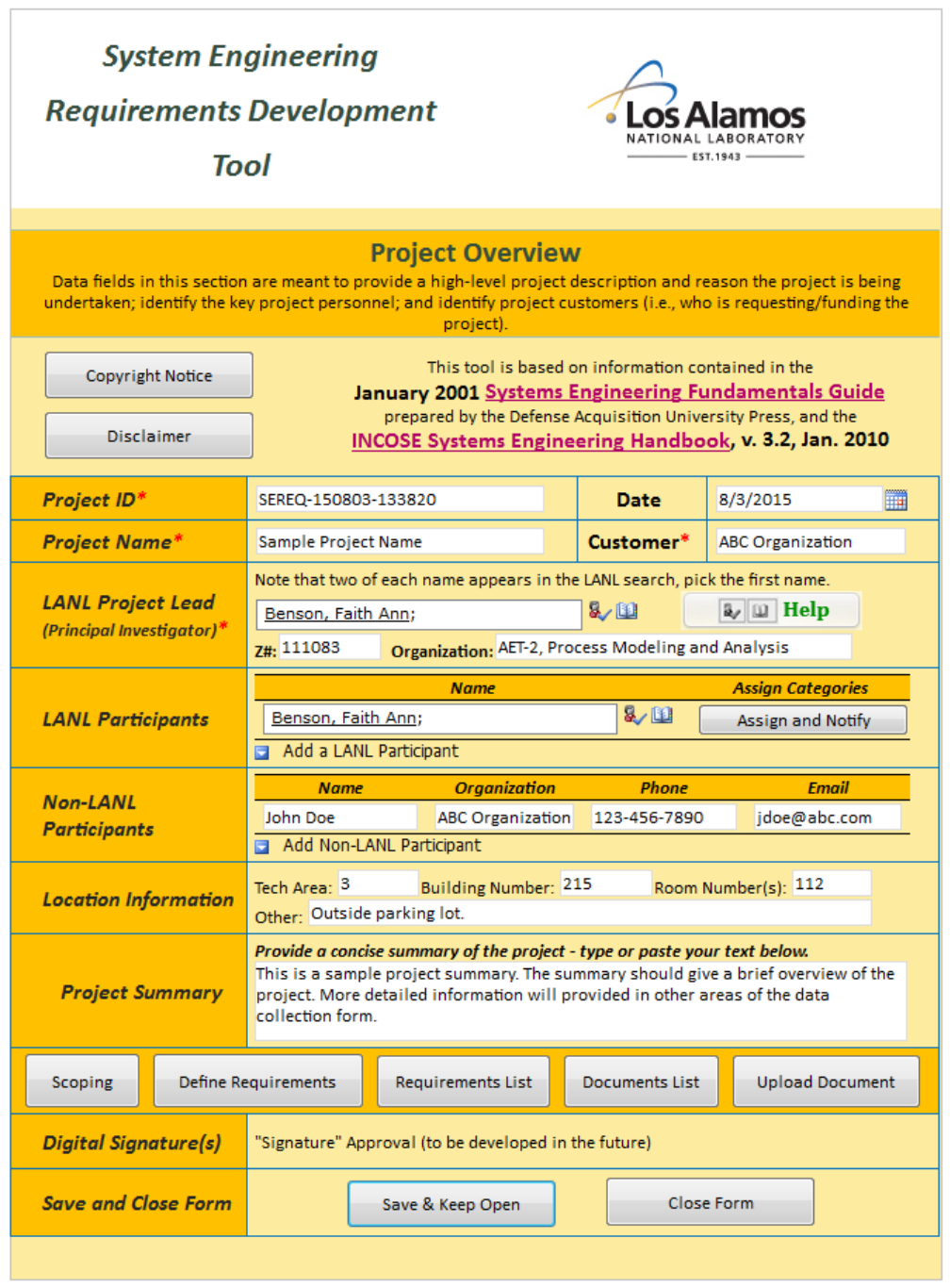

Figure 1. Project Overview Screen 


\subsection{Scoping}

This section is where the user begins to describe the key activities of the project and their associated products. This section is used to describe "what" the project must accomplish. For example, "provide the ability to test hardware." This information will be used to guide more specific "hows" in the Define Requirements section. The various sections include Mission Needs/Goals, Constraints/Expectations, Measures of Effectiveness, Operational Scenarios, System Boundaries, Interfaces, Modes of Operation, Technical Performance Measures, Life Cycle Process, Physical Characteristics, and Human Systems Integration.

\subsubsection{Section "Home" Pages}

Each section has its own "Home” page that contains section description and buttons that open the data entry form, upload related documentation, or close the form. (See Figure 2.) Definitions and explanations as to the type of data to be entered are contained throughout the tool. Some sections include "Help" buttons for further information or instruction.

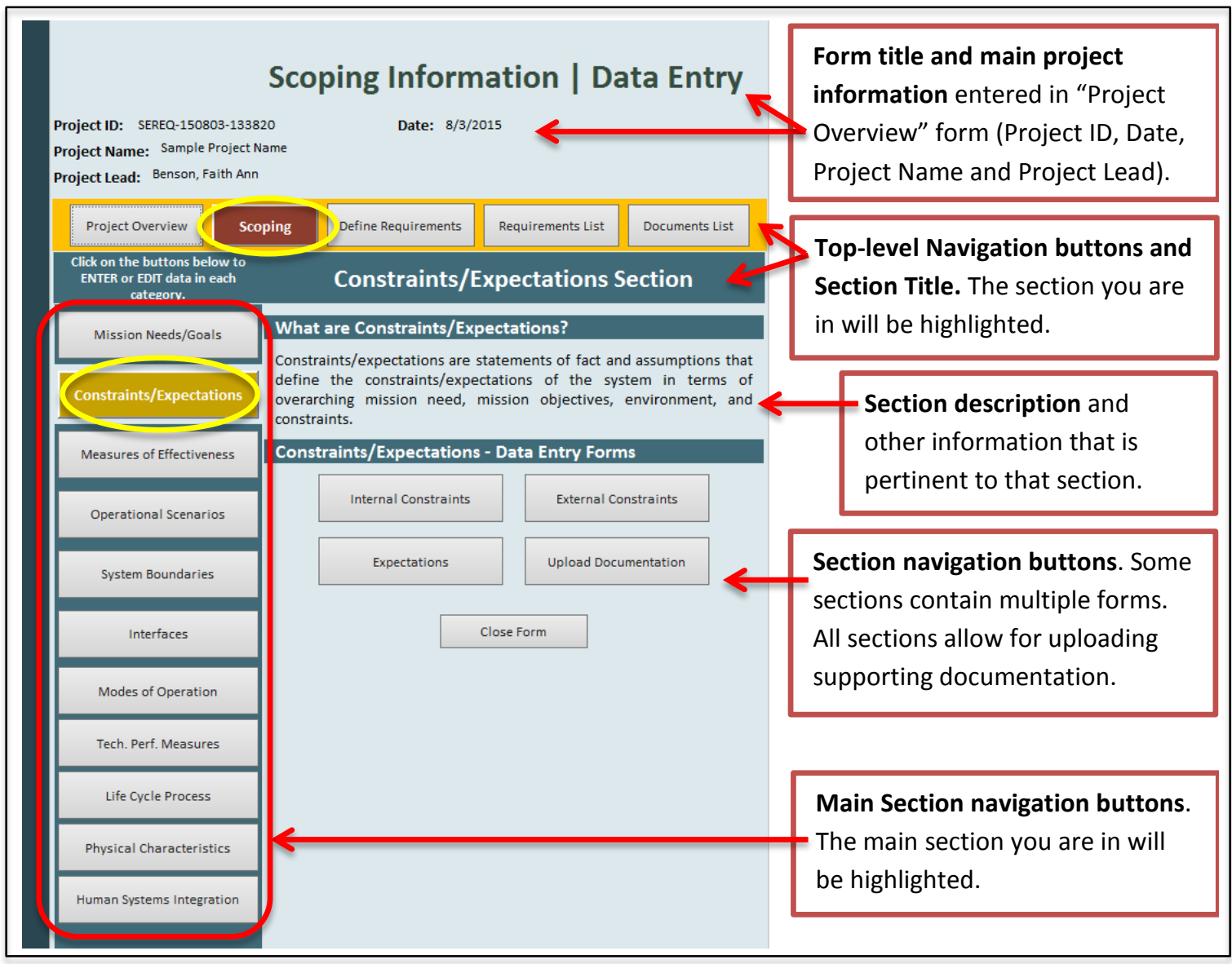

Figure 2. Example of a Section "Home" Page

\subsubsection{Scoping Data Entry Forms}

Each main section has at least one data entry form (some sections have more). This is where specific data related to that section is entered. This information is automatically transferred to the 
Define Requirements section where it will be displayed and used to define specific requirements. (See Figure 3.)

NOTE: The "Constraints/Expectations" section has extra layers of data entry forms in both "Scoping" and "Define Requirements" sections, but the navigation clues such as highlighted navigation buttons, section title bars, and explanation/

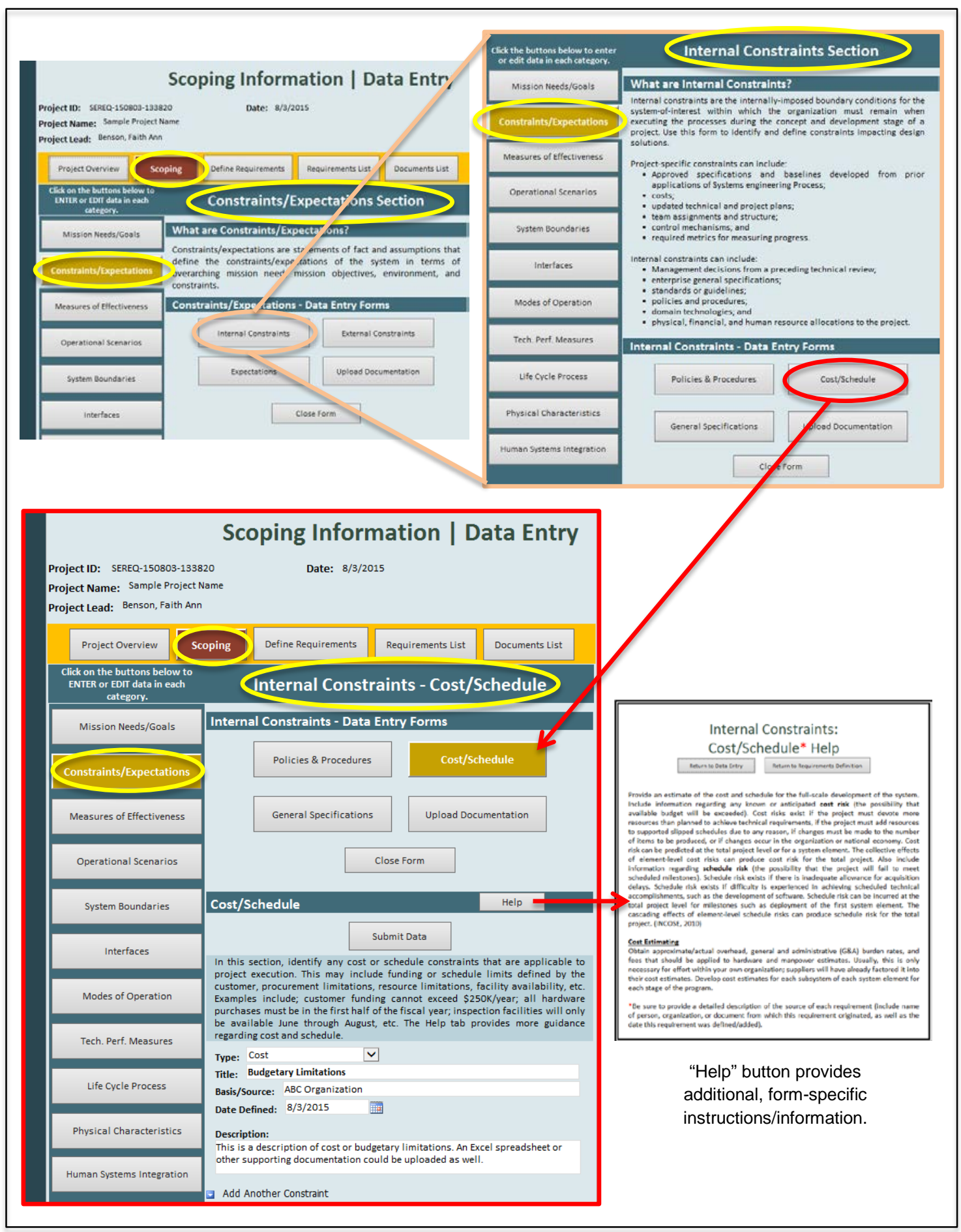

Figure 3. Example of Multiples Layers of Form Views. 
descriptions are the same throughout.

By clicking on the link at the bottom of every data entry form, additional information can be

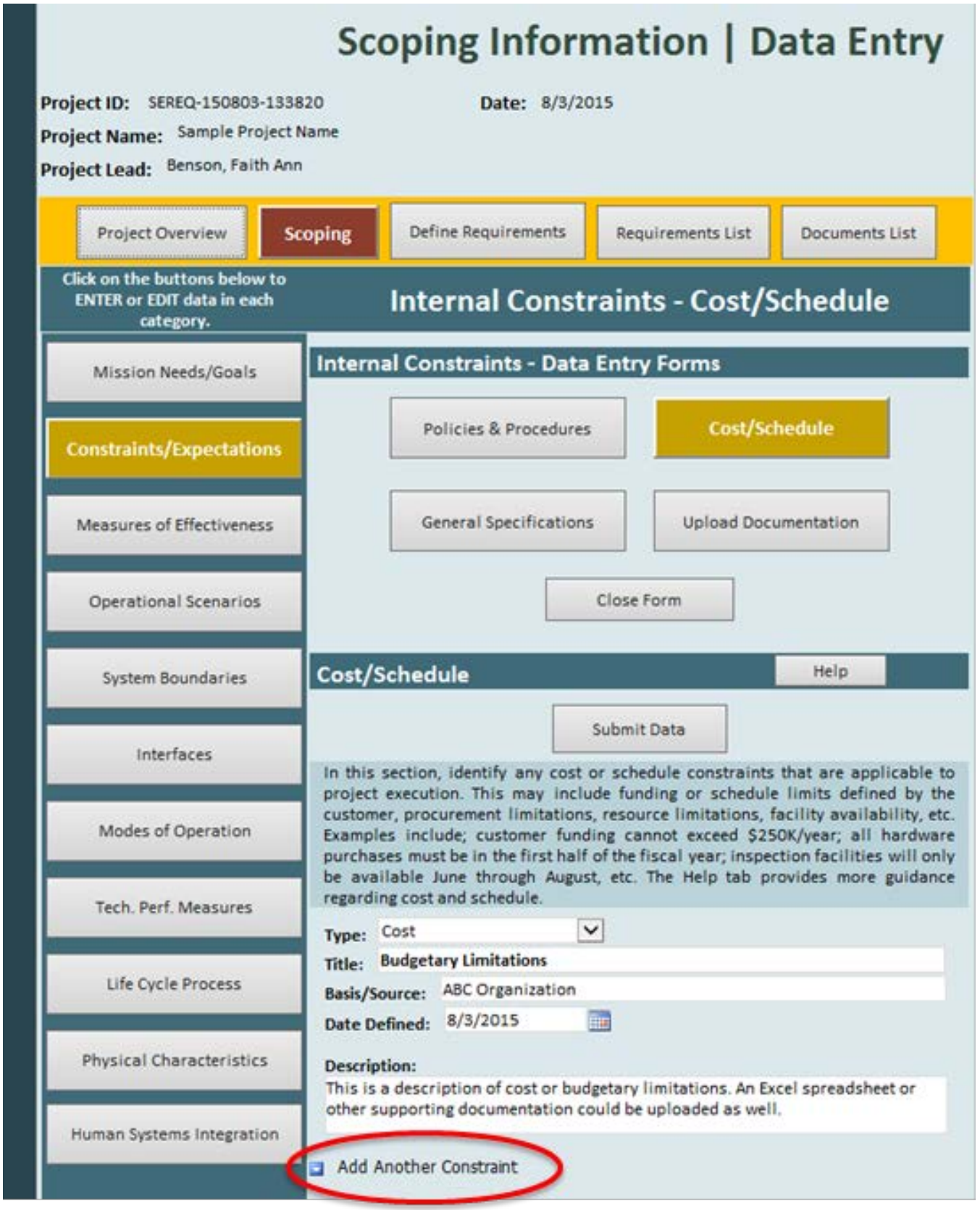

Figure 4. All Data Entry Forms have the option to add additional information.

added. (See Figure 4.) 


\subsection{Uploading Documents}

The user can upload supporting documentation by clicking on any of the "Upload

Documentation" buttons and then clicking on "Click here to attach a file." Additional documents can be uploaded by clicking "Upload another document.” (See Figure 5.)

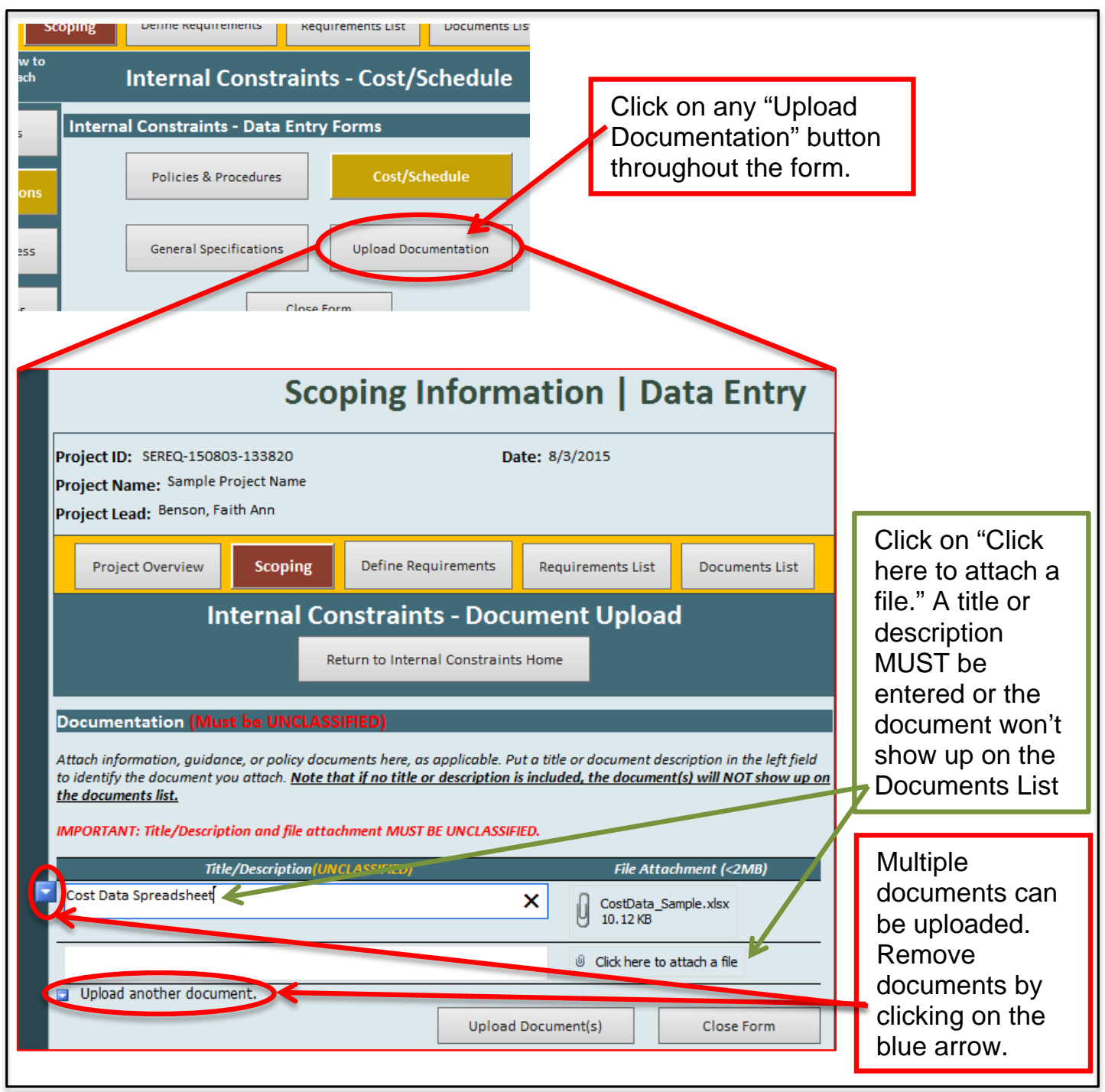

Figure 5. Uploading Documents

All uploaded documents will be displayed on the Documents List (see ). Note that if a title or description is not entered when the document is uploaded, the document will not display on the Documents List. All uploaded documents MUST BE UNCLASSIFIED. 


\section{Documents List}

\begin{tabular}{|c|c|c|c|c|}
\hline Project Overview & Scoping & Define Requirements & Requirements List & Documents List \\
\hline
\end{tabular}

Project ID: SEREQ-150803-133820

Date: $8 / 3 / 2015$

Project Name: Sample Project Name

Project Lead: Benson, Faith Ann

\section{Supporting Documentation List*}

If nothing shows below, no documents have been uploaded.

\section{Close Form}

CostData_Samplexdsx Micros oft Excel Worksheet $10.1 \mathrm{~KB}$

This is another test document

Testing the Form.doo Microsoft Word Document $49.3 \mathrm{~KB}$

\section{Internal Constraints Documents}

Cost Data Spreadsheet

Uploaded documents are only located within the InfoPath form but the user can click on the blue paperclip icon that pops up next to the desired document when the user clicks on the document icon and download a copy to their desktop. (See .)

\begin{tabular}{|c|c|c|}
\hline iternal Con & & \\
\hline Cost Data Spreadsheet & 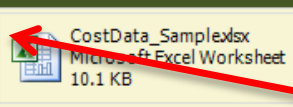 & $\begin{array}{l}\text { Click the paperclip } \\
\text { next to the }\end{array}$ \\
\hline This is another test document & $\begin{array}{l}\text { Testing the Form.doc } \\
\text { Microsoft Word Document } \\
49.3 \mathrm{~KB}\end{array}$ & $\begin{array}{l}\text { download a copy to } \\
\text { your desktop. }\end{array}$ \\
\hline
\end{tabular}

\subsection{Define Requirements}

This section is where the user will have the opportunity to define specific requirements. The main sections such as Mission Needs/Goals, Constraints/Expectations, Measures of Effectiveness, Operational Scenarios, etc., are duplicated here and the navigation clues are the same as in the "Scoping" section. 
In this section, if information was previously entered in the corresponding "Scoping” section, the information will be displayed and the user can define specific requirements based on that information (see ). If no information was entered for a specific section, the note "If no data was entered in this section, nothing will show below (see ).

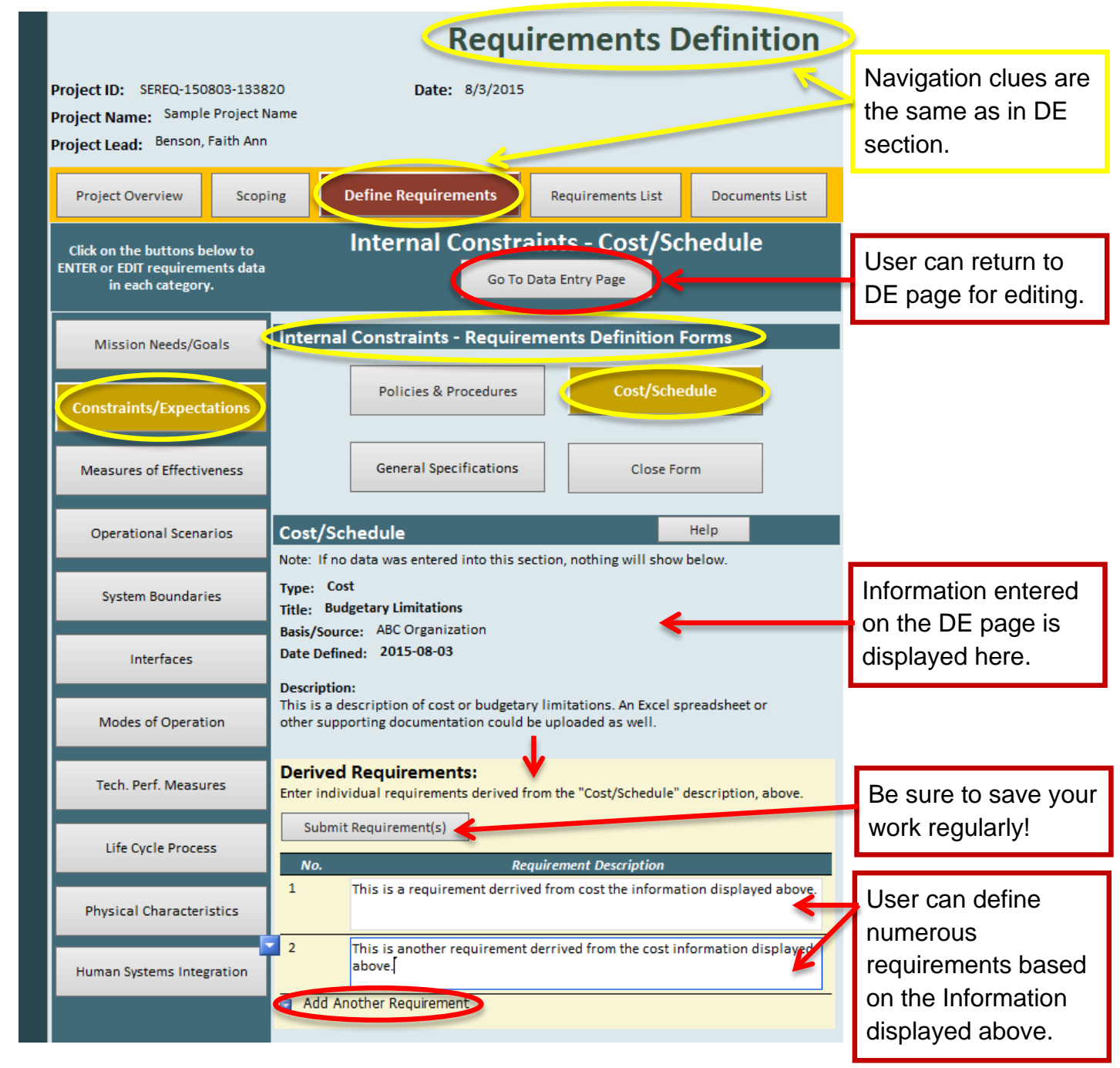

Figure 6. Example of "Requirements Definition" Page

\subsection{View Requirements}

At any time throughout the process the user can view the requirements that have been defined by clicking on the "View Requirements" button. 


\section{View Requirements}

\begin{tabular}{|l|l|l|l|l|l|}
\hline Project Overview & Define Requirements & View Requirements \\
\hline
\end{tabular}

Project ID: SEREQ-150803-133820

Date: $8 / 3 / 2015$

Project Name: Sample Project Name

Project Lead: Benson, Faith Ann

Add Requirement

Save and Keep Form Open

Close Form

Type: Cost

Title: Budgetary Limitations

CS- 1

This is a requirement derrived from cost the information displayed above.

CS-2

This is another requirement derrived from the cost information displayed above.

Save and Keep Form Open

Close Form

If you encounter issues not addressed by this user guide, please contact your account manager for additional support. 


\section{Exporting Data}

The purpose of this tool is to provide the user with a means of defining and devolving project requirements and capturing this information in a structured format. This information is also a valuable resource when discussing the project. To further enhance the value of the information input into the tool, two options are provided to export the data. First, An MS Word document is automatically generated for each project. This document is continually updated as information is input into the tool. The Word documents are saved to a separate document library in SharePoint. These Word documents can be downloaded and saved to the user's computer.

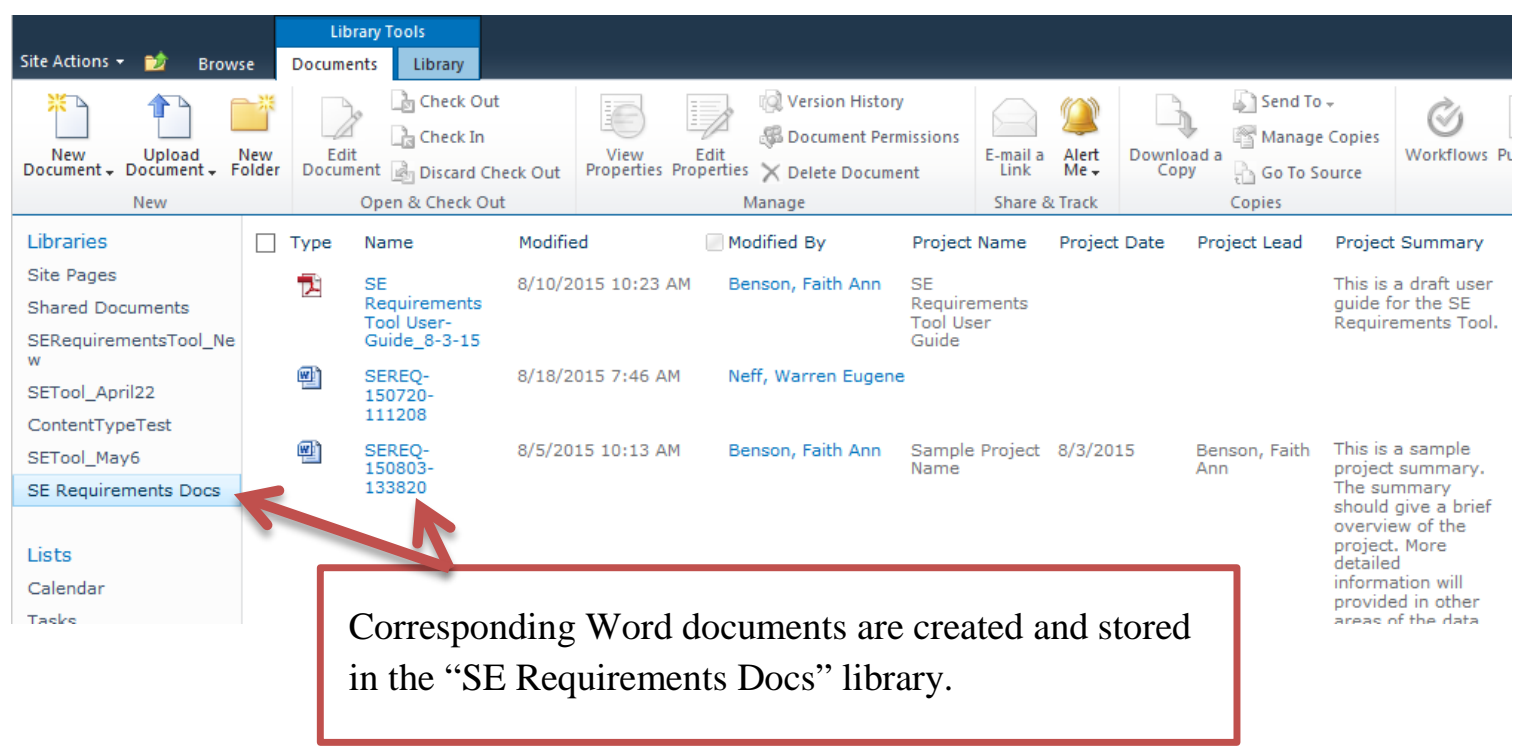

Second, the project information can also be exported into Excel from the SharePoint Library and saved to a local computer. When in the form library, click on the "Library" tab. Then click "Export to Excel."

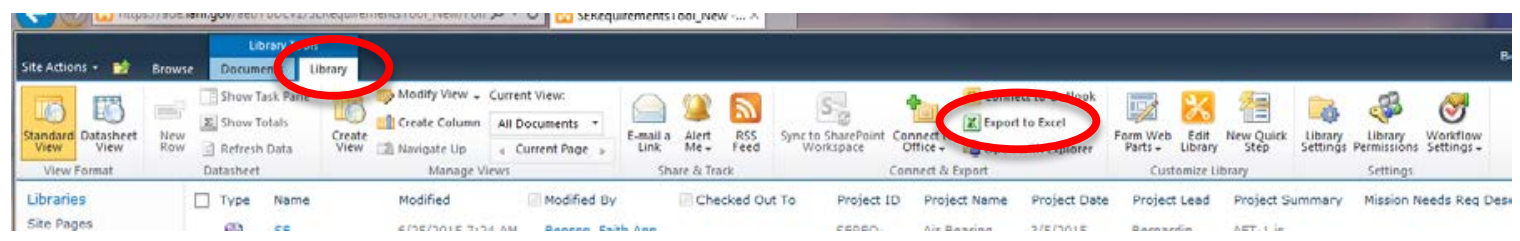

Note that the data from ALL of the documents in the library will be exported into an Excel document (one InfoPath form per line). There is no way at this time to export the data from only one InfoPath form into Excel. However, once downloaded and saved to the user's computer, unnecessary rows can be deleted. 